\title{
Signal and Noise Modeling in Confocal Laser Scanning Fluorescence Microscopy
}

\author{
Gerlind Herberich ${ }^{1}$, Reinhard Windoffer ${ }^{2, \star}$,

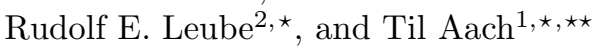 \\ 1 Institute of Imaging and Computer Vision, RWTH Aachen University, Germany \\ 2 Institute of Molecular and Cellular Anatomy, RWTH Aachen University, Germany
}

\begin{abstract}
Fluorescence confocal laser scanning microscopy (CLSM) has revolutionized imaging of subcellular structures in biomedical research by enabling the acquisition of 3D time-series of fluorescently-tagged proteins in living cells, hence forming the basis for an automated quantification of their morphological and dynamic characteristics. Due to the inherently weak fluorescence, CLSM images exhibit a low SNR. We present a novel model for the transfer of signal and noise in CLSM that is both theoretically sound as well as corroborated by a rigorous analysis of the pixel intensity statistics via measurement of the $3 \mathrm{D}$ noise power spectra, signal-dependence and distribution. Our model provides a better fit to the data than previously proposed models. Further, it forms the basis for (i) the simulation of the CLSM imaging process indispensable for the quantitative evaluation of CLSM image analysis algorithms, (ii) the application of Poisson denoising algorithms and (iii) the reconstruction of the fluorescence signal.
\end{abstract}

Keywords: Fluorescence microscopy, Confocal laser scanning microscopy, Model, Noise, Pixel intensity statistics.

\section{Introduction}

4D (3D+time) live cell fluorescence imaging by means of CLSM has become a widely used tool in cell biology for the analysis of the dynamics of subcellular structures. To avoid phototoxicity, the excitation laser power has to be kept in a low range. In consequence, the fluorescence signal is very weak and the SNR is low. Fig. 1b shows a typical CLSM slice image, depicting fluorescently-labeled keratin intermediate filaments, which are subcellular protein fibers that build an essential part of the cytoskeleton in epithelial cells, illustrating how much CLSM images are corrupted by noise.

Noise reduction is therefore a crucial preprocessing step to be applied before the image analysis. To perform a sound denoising a noise model is needed. An extended model describing signal and noise transfer in CLSM which does not only

\footnotetext{
* The authors gratefully acknowledge funding by the German Research Council (DFG, AA5/5-1, LE566/18-1, WI1731/8-1).

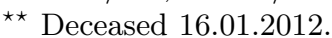




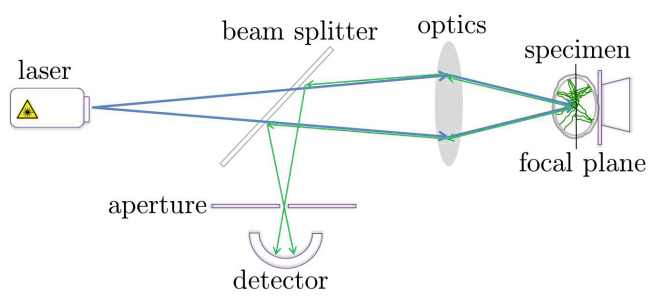

(a) CLSM beam path

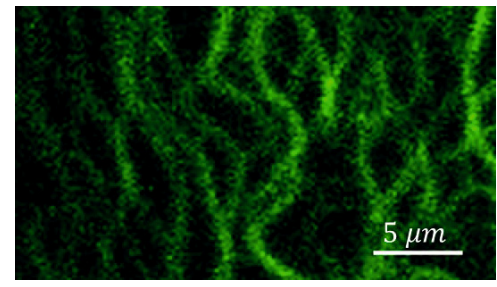

(b) Noise in CLSM data

Fig. 1. CLSM imaging: (a) Scheme of the beam path in a confocal laser scanning microscope and (b) Noise in CLSM images: Image section showing fluorescently-labeled keratin intermediate filaments, subcellular protein fibers, that appear in the image as curvilinear structures. Their granular appearance is due to noise.

account for the system noise but also for the randomness of the fluorescence input signal further enables the simulation of CLSM imaging so that synthetic images may be generated for the quantitative evaluation of image analysis algorithms for CLSM. Furthermore, such an extended model can serve for the reconstruction of the fluorescence input signal. The latter is related to the concentration of fluorescently-labeled proteins such that quantitative spatiotemporal analyses of the protein-of-interest can be performed.

Few models have been proposed for the noise in CLSM images as well as for the pixel intensity statistics. It is often assumed, that due to the photon-counting process in CLSM, the noise follows a Poisson distribution [1. Denoising methods for CLSM that rely on this assumption have been designed [2] [3. For the pixel intensity statistics in CLSM images, a mixture model [4] has been proposed recently, claiming that the pixel intensity statistics follow a linear mixture of a discrete normal distribution and a negative binomial distribution. The discrete normal distribution serves as a model for additive electronic noise while the negative binomial distribution, also known as Gamma-Poisson distribution, accounts for the fact that the photon-counting process at the detector described by the Poisson model depends itself on a random variable - namely the random fluorescence field after smoothing by the CLSM point-spread-function (PSF). The distribution of the latter is approximated by a Gamma distribution by simulation 4. Further related work on pixel intensity statistics includes a Poisson approximation to the noise of the integrating detectors 5] where the pixel intensity statistics are modeled as two cascaded Poisson processes and a linear mapping which accounts for the offset and gain of the analog-to-digital conversion.

We found that all of the above models did not fit well to our data (Fig. [5). Instead of performing a simulation of the input such as is done in [4, we provide a theoretically derived model which is substantiated by measurements of signaldependence, spatial-frequency-dependence and distribution of the pixel intensity statistics. 


\section{Image Formation}

In fluorescence CLSM, subcellular structures are labeled with fluorescent dyes called fluorochromes. The structure to be imaged can thus be considered as a spatial fluorochrome distribution. A 3D image of such a specimen is acquired as follows (Fig. 17): A laser is focused by the optics onto a point within the specimen (excitation beam path). Any fluorochromes present at that position randomly emit photons in all directions. As the fluorescence emitted by an excited fluorochrome has a longer wavelength than the excitation light (Stokes' law) the fluorescence photons are deflected at the dichroic beam splitter towards the detector, a photomultiplier tube (PMT). To mask fluorescence that originates from structures that are out-of-focus, an aperture is placed in front of the detector (detection beam path). By moving the focal point, the whole focal plane is scanned point-wise yielding a $2 \mathrm{D}$ image which represents a section of the specimen. This process is repeated at different depths of the $3 \mathrm{D}$ specimen such that it is imaged in a stack of $2 \mathrm{D}$ slices forming a $3 \mathrm{D}$ image.

For a better understanding of the way the fluorescence signal and the noise are transferred through the microscope system, in the following, we detail the single pixel acquisition process, i.e. the detection beam path, as illustrated in Fig. 2. Note that throughout the description the wavelength-dependency of the quantities involved is not mentioned explicitly for reasons of simplicity.

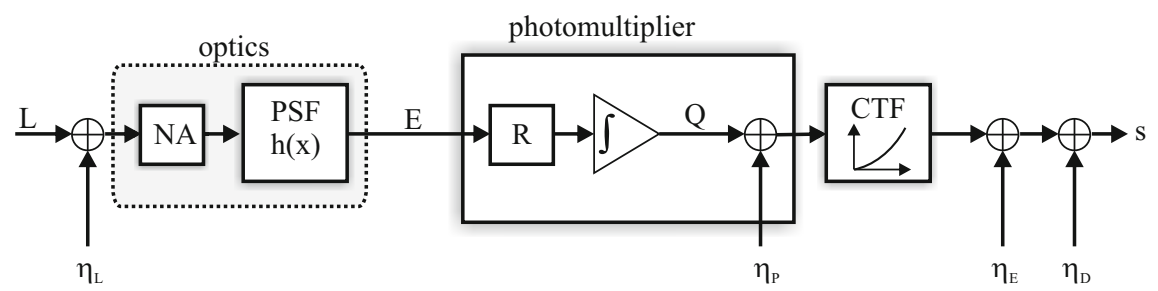

Fig. 2. Block diagram of the single pixel acquisition process in a confocal laser scanning microscope: See main text for explanation

The emission photon flux from a single fluorescent molecule depends on the excitation photon flux, the quantum yield of the fluorochrome and its molecular cross-section. It gives the number of photons emitted per unit time. However, not only a single fluorescent molecule contributes to the signal formation of one pixel, but rather all fluorescent molecules contained in the sensing volume form the input to the detection beam path, the radiant flux $L$. The number of fluorescent molecules in the sensing volume is determined by the fluorochrome concentration. The sensing volume is defined by the effective support of the PSF of the excitation beam path: It is the small volume within the specimen, that is illuminated by the laser during the pixel time.

Due to the quantum nature of light and electric charge, the emission of particles such as photons or electrons is associated with an uncertainty referred 
to as shot noise. According to this random process, photons are emitted in all spatial directions. Only a small fraction - the effective fraction - of them are collected by the microscope objective. Its numerical aperture NA is a measure for its acceptance cone. An objective with NA = 1.4 will capture about $30 \%$ of all photons. These photons pass the optics. The resulting radiant flux or radiant power $E$ specifies the number of photons per unit time arriving at the detector where they are integrated over the pixel dwell time $d t$. The detector responsivity $R \in[0,1]$ describes its quantum efficiency, i.e. how efficient incident photons cause photoelectrons to emit from the photocathode as a consequence of the photoelectric effect. Because of the quantum nature of electric charge, this process is subject to shot noise $\eta_{P}$. The electric field between photocathode and anode in the PMT accelerates the photoelectrons on their way to the anode while striking several dynodes where further electrons are emitted due to secondary emission. In this way, the number of electrons is multiplied and the accumulation of charge at the anode forms the detected signal which is largely amplified compared to the weak fluorescence radiant power arriving at the detector. The signal then passes an analog-to-digital converter (ADC), defining the final dynamic range of the output signal. It is sampled and quantized such that electronic noise $\eta_{E}$ and quantization noise $\eta_{D}$ are added to the signal. PMT gain, ADC offset, ADC gain and possible non-linearities in e.g. the electronics or nonlinear quantum efficiencies are summarized in the camera transfer function (CTF) 6]. The resulting noisy pixel intensity is called $s$.

\section{Measurements}

The pixel intensity statistics is the only quantity we can actually measure to corroborate our model derived in Section 4 with data. To measure signal-dependence, spatial-frequency-dependence and distribution of the pixel intensity statistics, under the assumption that all noise processes in the imaging chain are ergodic, we have sought specimens with a spatially homogeneous fluorochrome distribution to obtain a homogeneous input to the imaging chain. To this end, we prepared sodium fluorescein dilution series with known concentrations ranging from $5 \mu \mathrm{g} / \mathrm{ml}$ to $200 \mu \mathrm{g} / \mathrm{ml}$. Sodium fluorescein is a water soluble fluorochrome that has been proposed for fluorescence calibration [7]. Following [7] we stabilized the pH at 9.5 using borate buffer. Each specimen then yields a homogeneous fluorochrome distribution with a radiant power depending on the fluorescein concentration. At room temperature, from each specimen, we acquired a 16-bit 3D image stack using a Zeiss LSM710 with the following parameters: Excitation laser from the Argon line (wavelength of emission maximum: $488 \mathrm{~nm}$ ), lateral voxel size $d x=d y=24 \mathrm{~nm}$, interslice distance $d z=11 \mathrm{~nm}, 63 \times$ oil-immersion objective with numerical aperture $\mathrm{NA}=1.4$, pixel dwell time $d t=1.58 \mu \mathrm{s}$, pinhole diameter $49 \mu \mathrm{m}$, PMT gain 911, ADC gain $K_{d}=1$, ADC offset $K_{0}=0$.

To investigate whether the pixel intensity variance is signal-dependent, we measured sample variance and sample mean slicewise (Fig. 3a) over $1.8 \cdot 10^{8}$ samples from concentrations which spanned a range from 20 to $200 \mu \mathrm{g} / \mathrm{ml}$ at 


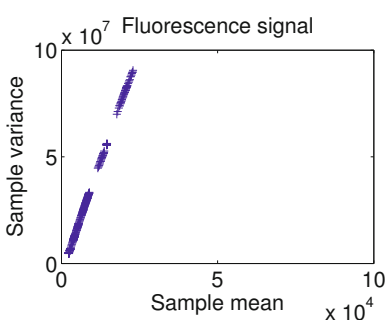

(a)

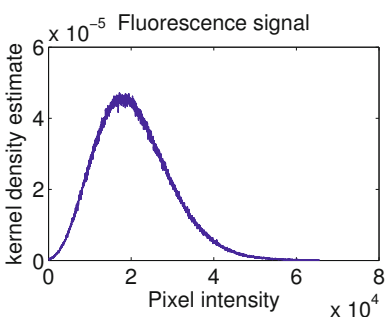

(b)

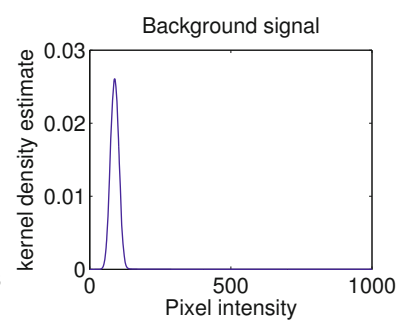

(c)

Fig. 3. Fluorescence signal: (a) signal-dependence of variance and (b) kernel density estimate of pixel intensity distribution. Background signal: (c) kernel density estimate of intensity distribution. Note that the gaps in plot (a) are due to a limited number of fluorescein concentrations used for the analysis.

$20 \mu \mathrm{g} / \mathrm{ml}$ increments and from $5 \mu \mathrm{g} / \mathrm{ml}$ and $10 \mu \mathrm{g} / \mathrm{ml}$. Note that the slope is not equal to one as would be the case for samples of a Poisson distribution where the variance equals the expected value.

As estimate of the intensity distribution, for each fluorescein image, we computed a kernel density estimate from the pixel intensities. We used the Epanechnikov kernel with a width of 10 . Fig. 3 b shows a kernel density estimate of $2 \cdot 10^{6}$ samples drawn from a 3D image of a $180 \mu \mathrm{g} / \mathrm{ml}$-concentration specimen and Fig. 3. a density estimate from $8 \cdot 10^{5}$ samples of a $3 \mathrm{D}$ image acquired without a specimen to measure the electronic noise.

We have estimated the 3D noise power spectrum (NPS) by periodogram averaging (Bartlett method). Fig. 4 shows the results of averaging over $700 x y$ - and $x z$-slices of size $100 \times 100$ respectively.
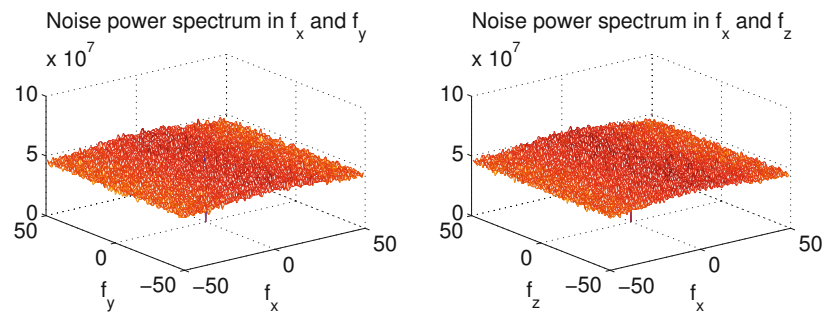

Fig. 4. NPS estimates in $x, y$ and $z$ : White spectrum in $f_{y}$ and $f_{z}$ and a slight increase in low spatial frequencies in $f_{x}$ due to linewise scanning. (The sharp negative peak at $\mathbf{f}=0$ is due to subtraction of the sample mean prior to periodogram averaging.).

We can draw the following conclusions: As expected, the electronic noise $\eta_{E}$ can be modeled as additive Gaussian noise and is of negligible amplitude. Quantization noise $\eta_{D}$ may be neglected as it exhibits an amplitude smaller than half a quantization level. Obviously, the dominating noise sources are the fluorescence noise $\eta_{L}$ and/or the PMT noise $\eta_{P}$. This conclusion is confirmed by 
the measurement of the signal-dependence which indicates by its slope that the noise samples have largely been amplified. The 3D NPS further indicates that the power of $\eta_{P}$ must be superior to that of the fraction of $\eta_{L}$ that arrives at the detector: White noise $\eta_{L}$ passing through optics becomes colored noise with an NPS shaped by the squared magnitude of the Fourier transform of the PSF. As the measured NPS is not colored, the power of $\eta_{P}$ must be larger.

\section{Model}

The random fluorescence signal is the input to the imaging system. In the case of weak signals, the law of rare events holds and the uncertainty of the fluorescence photon emission can be modeled by a Poisson distribution describing the probability to find $k$ successes (i.e. photon emissions) in a given time interval. Let $X_{L}$ be a discrete random variable, $X_{L} \sim \mathcal{P}(L)$ with probability mass function (pmf) $P_{X_{L}}(k)=\frac{L^{k} e^{-L}}{k !}, k \in \mathbb{N}, L \in \mathbb{R}^{>0}$. Recall that for a Poisson distribution, the variance equals the expected value: $\operatorname{Var}\left(X_{L}\right)=L$. Hence, to model this as additive noise, we rewrite $\eta_{L}=k-L$ as depicted in Fig. 2 ,

In the following, we describe step by step how the random fluorescence signal is transferred through each of the blocks in the diagram Fig. 2 and where it is corrupted by noise.

1) NA: From $k$ emitted photons, only a small fraction $l$ are collected by the microscope objective: The random variates $k$ become new random variates $l=$ $f_{1}(k)$ of the discrete random variable $X_{\mathrm{NA}}$, according to $f_{1}(x)=a_{1} x, a_{1} \in[0,1]$. $\operatorname{Var}\left(X_{\mathrm{NA}}\right)=a_{1}^{2} \operatorname{Var}\left(X_{f}\right)$.

2) PSF: The $l$ collected photons are then filtered by the PSF which corresponds to a convolution of the effective fraction of the spatial fluorochrome distribution with the PSF $h(\mathbf{x})$ of the microscope, $\mathbf{x}=(x, y, z)^{T}$ being the spatial coordinates. Estimating the PSF using the fluorescein images in a similar fashion as in [8] is not possible because of the PMT noise $\eta_{P}$. We thus base our modeling upon a PSF description by a linear shift-invariant (LSI) lowpass. As an example, the 3D anisotropic Gaussian approximation [9] leads to Gaussian parameters $\sigma_{x}=\sigma_{y}=78.5 \mathrm{~nm}$ and $\sigma_{z}=294 \mathrm{~nm}$ for the paraxial model given our imaging parameters. Calculating how the pmf changes due to transfer of the random variates through an LSI-system is a nontrivial task. We approximate this by considering how the linear and quadratic mean change: The linear mean becomes $E\left(X_{E}\right)=H(0) E\left(X_{\mathrm{NA}}\right), H(\mathbf{f})$ being the Fourier transform of $h(\mathbf{x})$ and $\mathbf{f}=\left(f_{x}, f_{y}, f_{z}\right)^{T}$ being the spatial frequencies. The variance of zero-mean white noise after filtering is $\operatorname{Var}\left(X_{E}\right)=\operatorname{Var}\left(X_{\mathrm{NA}}\right) \sum_{\mathbf{i}} h^{2}(\mathbf{i})$. In consequence, for an LSIlowpass PSF model, the mean remains unchanged by filtering and the variance is highly reduced. Hence, we approximate the pmf after filtering by a mapping of the input pmf that leaves the mean unchanged while decreasing the variance. This yields new variates $m=f_{2}(l)$ with $f_{2}(x)=a_{2}\left(x-E\left(X_{\mathrm{NA}}\right)\right)+E\left(X_{\mathrm{NA}}\right)$, $a_{2}^{2}=\sum_{\mathbf{i}} h^{2}(\mathbf{i})$.

3) $R$ : The detector responsivity $R$ leads to new random variates $n=f_{3}(m)$ of the random variable $X_{R}, f_{3}(x)=a_{3} x, a_{3} \in[0,1]$ and $\operatorname{Var}\left(X_{R}\right)=a_{3}^{2} \operatorname{Var}\left(X_{E}\right)$. 
To summarize the mappings above, $n=f(k)$ according to $f(x)=y=$ $a_{2} a_{3}\left(a_{1} x-E\left(X_{\mathrm{NA}}\right)+\frac{E\left(X_{\mathrm{NA}}\right)}{a_{2}}\right)$. The variance has become

$$
\operatorname{Var}\left(X_{R}\right)=a_{3}^{2} \sum_{\mathbf{i}} h^{2}(\mathbf{i}) a_{1}^{2} L=a_{1}^{2} a_{2}^{2} a_{3}^{2} L=c L
$$

and $c \ll 1$ because $a_{1}$, describing the acceptance cone of the optics, is very small and $a_{2}^{2} \ll 1$ for the Gaussian PSF model introduced above. Hence, even for ideal detector responsivity $R=1, c \ll 1$ and $\operatorname{Var}\left(X_{R}\right)$ becomes negligible as $L$ decreases or rather is in the low signal range. Note that this assessment is in accordance with our NPS measurement Fig. 4. The conclusion we can draw is that $Q$ can be approximated as deterministic signal $Q=a_{1} a_{3} L$.

4) PMT: The PMT shot noise can be modeled by a Poisson distribution: $X_{Q} \sim \mathcal{P}(Q)$ according to the pmf $P_{X_{Q}}\left(k_{q}\right)=\frac{Q^{k_{q}} e^{-Q}}{k_{q} !}$.

5) CTF: The CTF accounts for the signal amplification in the PMT, the rescaling in the $\mathrm{ADC}$, the $\mathrm{ADC}$ offset, dark current and possible non-linearities in the imaging chain. We model it using a gamma curve to obtain a simple model of possible non-linearities which still contains a linear transform as special case. The random variates $k_{q}$ are transformed into new samples $k_{s}=f_{C T F}\left(k_{q}\right)$ according to $y=f_{C T F}(x)=\alpha+\beta x^{\gamma}, \alpha \geq 0$. The substitution $f_{C T F}^{-1}(y)=\varphi(y)=\left(\frac{y-\alpha}{\beta}\right)^{1 / \gamma}$ with the continuously differentiable function $\varphi(y)$ gives the new pmf

$$
P_{X_{s}}\left(k_{s}\right)=\frac{d \varphi}{d y} P_{X_{Q}}\left(k_{q}\right)=\frac{Q^{\left(\frac{k_{s}-\alpha}{\beta}\right)^{1 / \gamma}} e^{-Q}}{\left(\left(\frac{k_{s}-\alpha}{\beta}\right)^{1 / \gamma}\right) !} \frac{1}{\gamma \beta}\left(\left(\frac{k_{s}-\alpha}{\beta}\right)^{\frac{1}{\gamma}-1}\right) .
$$

As $\eta_{E}$ and $\eta_{D}$ are negligible, the noisy signal $s=k_{s}$ and the pixel intensity statistics follow $P_{X_{s}}\left(k_{s}\right)$.

To fit this model to our data, we used the Stirling approximation for factorial computation. Fig. 5 shows the estimated CTF with the parameters $\alpha=58.5$, $\beta=3028$ and $\gamma=1.08$ as fitting result. Fig. [5] shows two example distributions illustrating that our model provides a very accurate description of the data.
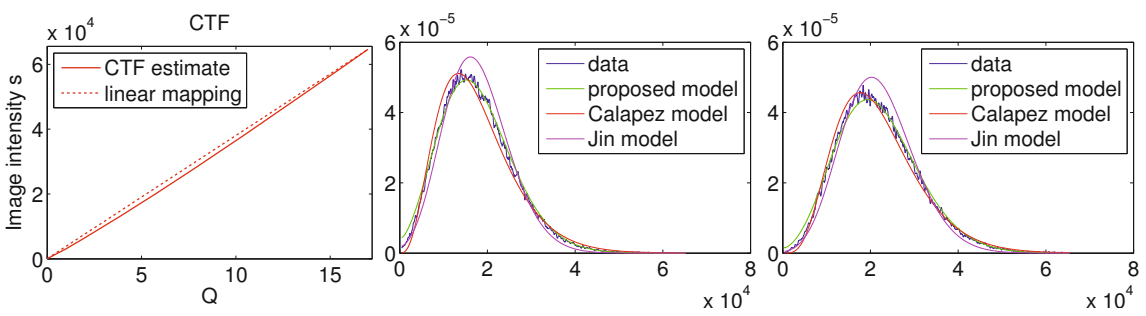

Fig. 5. Model fit. Left: Estimated CTF showing that the imaging system exhibits a slight non-linearity. A linear mapping is shown for comparison. Center and Right: The proposed model is plotted over the kernel density estimate. The Calapez model fit 4] and the Jin model fit [5] are shown for comparison. 
The Calapez model [4] and the Jin model [5] are shown for comparison. The mean absolute fitting error (MAE) over $2 \cdot 10^{6}$ samples confirms this result. The MAE of the proposed model is $8.04 \cdot 10^{-7}$ while the MAE of the Calapez model is $1.23 \cdot 10^{-6}$ and that of Jin model fit is $1.96 \cdot 10^{-6}$. Note that as $\eta_{E}$ is negligible, the Jin model fit corresponds to the fit of our model for $\gamma=1$ which equals a fit of the standard model of a linearly-scaled Poisson.

\section{$5 \quad$ Summary and Conclusions}

In the present work, we have a proposed a novel model for signal and noise transfer in CLSM. We have acquired homogeneous exposures from sodium fluorescein dilution series. These images served as basis for a rigorous analysis of the pixel intensity statistics via assessment of their distribution, signal-dependence and spatial frequency-dependence. These measurements illuminated the understanding of the imaging process and substantiate our new model which gives a description of the pixel intensity statistics derived from a Poisson process whose variates are mapped to the pixel intensity via the camera transfer function accounting for possible non-linearities in the system. It enables simulation of the CLSM image formation so that CLSM image analysis algorithms can quantitatively be evaluated. After inverse CTF, established Poisson denoising methods can be applied. Finally it forms the basis for a reconstruction of the input fluorescence signal. As the model fit is performed with data acquired from sodium fluorescein concentration series, the procedure can easily be repeated to determine the model parameters for other microscopes.

\section{References}

1. Sheppard, C.J.R., Gan, X., Gu, M., Roy, M.: Signal-To-Noise Ratio in Confocal Microscopes. In: Pawley, J.B. (ed.) Handbook of Biological Confocal Microscopy, 3rd edn. ch. 22, pp. 442-452. Springer (2006)

2. Rodrigues, I., Sanches, J.: Convex total variation denoising of poisson fluorescence confocal images with anisotropic filtering. IEEE TIP 20(1), 146-160 (2011)

3. Kervrann, C., Trubuil, A.: An adaptive window approach for poisson noise reduction and structure preserving in confocal microscopy. In: Proc. ISBI, pp. 788-791 (2004)

4. Calapez, A., Rosa, A.: A statistical pixel intensity model for segmentation of confocal laser scanning microscopy images. IEEE TIP 19(9), 2408-2418 (2010)

5. Jin, X.: Poisson approximation to image sensor noise. Master's thesis, University of Dayton, Ohio (2010)

6. Bell, A.A., Brauers, J., Kaftan, J.N., Meyer-Ebrecht, D., Böcking, A., Aach, T.: High dynamic range microscopy for cytopathological cancer diagnosis. IEEE JSTSP (Special Issue: Dig. Im. Proc. Techniques for Oncology) 3(1), 170-184 (2009)

7. Marti, G.E., et al.: Fluorescence calibration and quantitative measurement of fluorescence intensity; approved guideline. NCCLS 24(26) (2004)

8. Brauers, J., Aach, T.: Direct PSF estimation using a random noise target. In: IS\&T/SPIE Electronic Imaging. SPIE-IST, vol. 7537 (2010)

9. Zhang, B., Zerubia, J., Olivo-Marin, J.C.: Gaussian approximations of fluorescence microscope point-spread function models. App. Opt. 46(10), 1819-1829 (2007) 